\section{References}

1. Fan M, Sun D, Zhou T, Heianza Y, LV J, Li L, Qi L: Sleep patterns, genetic susceptibility, and incident cardiovascular disease: a prospective study of 385292 UK biobank participants. Eur Heart J 2020;41:1182-1189. DOI: 10.1093/eurheartj/ ehz849

2.Li X, Xue Q, Wang M, Zhou T, Ma H, Heianza Y, Qi L: Adherence to a Healthy Sleep Pattern and Incident Heart Failure: A Prospective Study of 408802 UK Biobank Participants. Circulation 2021;143:97-99. DOI: 10.1161/CIRCULATIONAHA.120.050792

3.Hering D, Wolf J, Chrostowska M, Narkiewicz K: Obesity and Obstructive Sleep Apnoea. In: Mancia G, Grassi G, Tsioufis KP, Dominiczak AF Rosei EA, editors. Manual of Hypertension of the European Society of Hypertension. CRC Press, 2019. DOI:10.1201/9780429199189-19

4. Benjafield AV, Ayas NT, Eastwood PR, Heinzer R, Ip MSM, Morrell MJ, Nunez CM, Patel SR, Penzel T, Pepin JL, Peppard PE, Sinha S, Tufik S, Valentine K' Malhotra A: Estimation of the global prevalence and burden of obstructive sleep apnoea: a literaturebased analysis. Lancet Respir Med 2019;7:687-698. DOl: 10.1016/S2213-2600(19)30198-5

5. Lombardi C, Pengo MF, Parati G: Systemic hypertension in obstructive sleep apnea. J Thorac Dis 2018;10:S4231-S4243. DOl: 10.21037/ jtd.2018.12.57
6. Ludka O, Stepanova R, Sert-Kuniyoshi F, Spinar J, Somers VK, Kara T: Differential likelihood of NSTEMI vs STEMI in patients with sleep apnea. Int J Cardiol 2017;248:64-68. DOI: 10.1016/j.ijcard.2017.06.034

7. Albuquerque FN, Calvin AD, Sert Kuniyoshi FH, Konecny T, Lopez-jimenez F, Pressman GS, Kara T, Friedman P, Ammash N, Somers VK, Caples SM: Sleep-disordered breathing and excessive daytime sleepiness in patients with atrial fibrillation. Chest 2012;141:967-973. DOI: 10.1378/chest.11-0975

8.Linz D, Brooks AG, Elliott AD, Nalliah CJ, Hendriks JML, Middeldorp ME, Gallagher C, Mahajan R, Kalman JM, McEvoy RD, Lau DH, Sanders P: Variability of Sleep Apnea Severity and Risk of Atrial Fibrillation: The VARIOSA-AF Study. JACC Clin Electrophysiol 2019;5:692-701. DOI: 10.1016/j.jacep.2019.03.005

9.Lachowska K, Gruchala M, Narkiewicz K, Hering D: Sympathetic Activation in Chronic Heart Failure: Potential Benefits of Interventional Therapies. Curr Hypertens Rep 2016;18:51. DOI: 10.1007/ s11906-016-0660-7

10.Leino A, Westeren-Punnonen S, Toyras J, Myllymaa S, Leppanen T, Yla-Herttuala S, MurajaMurro A, Kantanen AM, Autere J, Jakala P, Mervaala E, Myllymaa K: Acute stroke and TIA patients have specific polygraphic features of obstructive sleep apnea. Sleep Breath 2020;24:1495-1505. DOl: $10.1007 / \mathrm{s} 11325-019-02010-2$

\title{
Pathophysiology of Sleep Disordered Breathing
}

\section{JONAS SPAAK}

Karolinska Institutet, Department of Clinical Sciences, Danderyd Hospital, Division of Cardiovascular Medicine; and Danderyd University Hospital, Department of Cardiology, Stockholm, Sweden.

DOI:10.30824/2106-14

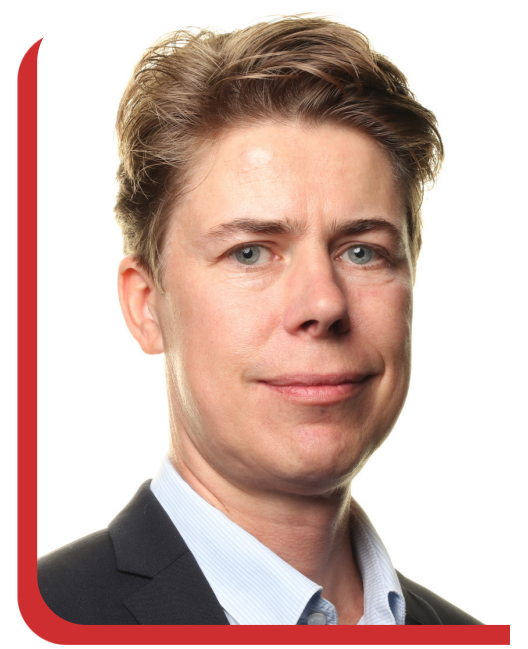

\section{Introduction}

Twenty years ago, it would have been easy to summarise the pathophysiology of sleep disordered breathing as repetitive nocturnal apnoea in obese persons causing sympathetic nerve system activation, hypertension and over time general cardiovascular disease, heart failure and stroke. The continuing studies in the field have added considerable knowledge but also many exceptions to this simplified description. Furthermore, despite the wealth of physiological understanding, the evidence for best treatment beyond weight loss and life-style modifications is limited. Part of the explanation for this is that most trials have excluded symptomatic patients, as it has been considered unethical not to offer them treatment. Nonetheless the strong association between sleep disordered breathing and obstructive sleep apnoea (OSA) in particular, 
and cardiovascular disease is well established. ${ }^{1,2}$ The term sleep disordered breathing include OSA, central sleep apnoea (CSA) and the combination of these (complex sleep apnoea). This short review will focus on the pathophysiology of OSA. For a complete account of sleep apnoea and cardiovascular disease the author recommend an excellent review by professor John Floras (the author's post-doc mentor). ${ }^{2}$

\section{Acute physiological effects of OSA}

Obstructive apnoea or hypopnea occur when the upper airway collapses completely or partially during sleep impeding inspiration. The cause of loss of airway-patency is extremely complex but is more common in overweight individuals, detailed in a review by Dempsey et al. ${ }^{3}$ Each obstruction triggers a cascade of events. ${ }^{3}$ The repetitive but failed inspiratory efforts markedly reduce the intrathoracic pressure, up to -60 $\mathrm{mmHg}$. This increases the transmural pressure difference over the large vessels including the aortic arch and the heart with the equivalent amount, since these are hydraulically connected with the extra-thoracic circulation and increase cardiac afterload. Ventricular interdependence impedes right ventricular filling despite an increased systemic venous return. Pulmonary and aortic baroreceptors (stretch receptors) sense the increased transmural pressure gradient and misinterpret this as an increase in blood pressure, and at the brain stem level inhibit sympathetic efferent discharge to the heart, kidneys, and resistance vessels. These combined effects lead to a reduction in heart rate and cardiac output, and temporarily reduce peripheral resistance and a drop in arterial blood pressure. This makes the circulatory system poorly prepared to handle the subsequent arterial oxygen desaturation and $\mathrm{CO} 2$ accumulation that now takes over and elicit sympathetic excitation and vasoconstriction, at the same time as the hypoxia increase efferent vagal tone. The desaturations may reach $60 \%$ $\mathrm{SaO} 2$ or lower. The apnoea usually terminates after 30-90 seconds due to arousal, that further stimulate sympathetic activation. In many patients this is, after a few deep breaths, repeated again and again, 30 to 60 times an hour. These effects do not occur to the same degree in all patients but represent the classical pathophysiology behind OSA (Table 1). ${ }^{3}$ It is important to note that the apnoea-induced sympathetic excitation carry-over also to an increased daytime activation, and that patients with heart failure handle these challenges less well. ${ }^{4}$

Table 1. Obstructive apnea: consequences of negative intrathoracic pressure

\begin{tabular}{l} 
Acute: atrial, ventricular, pulmonary, and thoracic vessel distension, increased afterload, increased \\
myocardial $\mathrm{O}_{2}$ demand, decreased coronary flow, reduced stroke volume, reflexive sympathetic nerve \\
firing and noradrenaline release \\
\hline $\begin{array}{l}\text { Chronic: increased risk for atrial fibrillation, atrial and ventricular hypertrophy or dilatation, thoracic } \\
\text { aortic dilatation }\end{array}$ \\
\hline Not detected by brachial blood pressure measurements \\
\hline Not affected by antihypertensive treatment \\
\hline Based on Floras, 2018.2
\end{tabular}

\section{OSA in hypertension}

It has been shown that the apnoea-induced sympathetic activation and mechanical effects over time cause endothelial dysfunction, and contribute to aortic stiffness. ${ }^{5}$ Concordant increases in efferent renal sympathetic nerve traffic stimulate both noradrenergic and renin-aldosterone mediated sodium and water retention. ${ }^{6}$ At the same time the disrupted sleep pattern have negative effects on glucose homeostasis and contribute to systemic inflammation. ${ }^{7}$ Over time these combined effects contribute to the development of hypertension. ${ }^{2}$
In line with this, the prevalence of hypertension in individuals with at least moderate OSA is higher even after adjustments for common comorbidities. For instance, the large HypnoLaus population survey $(n=2,121)$, after multivariable adjustment, demonstrated an odds ratio for hypertension prevalence of 1.6 for moderatesevere OSA (apnoea-hypopnea index $>20 / \mathrm{h}$ ). ${ }^{1}$ In one small cohort of patients with therapy-resistant hypertension the prevalence was as high as $83 \%{ }^{8}$

Despite this strong association, treatment of OSA with continuous positive airway pressure 
(CPAP) only reduce blood pressure on average $2 / 2 \mathrm{mmHg} .{ }^{9}$ Part of the explanation may be that our techniques to measure blood pressure, such as ambulatory blood pressure monitoring, fails to capture the transient blood pressure swings associated with repeated apnoea. Each intermittent measurement may trigger an arousal terminating any apnoea. Indeed, one study utilising continuous blood pressure measurements by digital photoplethysmography showed that therapeutic continuous positive airway pressure lowered average night-time and daytime blood pressure by $13 / 11$ and $10 / 11 \mathrm{~mm} \mathrm{Hg}$, respectively, in 36 randomized (58\% hypertensive) participants. ${ }^{10}$ Further support for a causative association is given in a recent meta-analysis including 5,550 participants showing a pooled prevalence of left ventricular hypertrophy (LVH) of $45 \%$ in the OSA population, with an odds ratio 1.70 for LVH in subjects with OSA as compared to controls. ${ }^{11}$ Still, it is an unanswered question why some patients with OSA appears to be protected and do not develop hypertension.

\section{OSA and cardiovascular diseases}

The association between OSA and several cardiovascular diagnoses is well established in population studies, but it is hard to elucidate the exact relationship since OSA often is recognised in relation to an event, and it is difficult to separate the effects of confounders and mediators, such as obesity and blood pressure. ${ }^{1}$ In brief, it may be advisable to consider OSA in all patients experiencing nocturnal cardiovascular events, such as stroke, angina, acute coronary syndrome or sudden cardiac death. ${ }^{2}$ The acute haemodynamic effects may also contribute to aortic strain and OSA should be considered in patients with aortic aneurysm. ${ }^{2}$ Atrial fibrillation is common in OSA patients, and is associated with increased risk for relapse after cardioversion or ablation. ${ }^{12}$ Anatomically the pulmonary vein area is responsible for the majority of atrial fibrillation, and is located just outside the stiff pericardium and hence susceptible to stretch during OSA induced pressure swings. In pulmonary hypertension, OSA, in the absence of any alternative cause, can be detected in $20-40 \%$ of individuals and is potentially directly caused by the von Euler-Liljestrand mechanism. ${ }^{13}$

OSA in heart failure is complex and deserve its own review article. The shared pathophysiology with increased neurohumoral activation and hypertension in addition to peripheral oedema and nocturnal rostral fluid shift contributes to a high prevalence of OSA in heart failure. ${ }^{6}$ In patients hospitalised with decompensated hear failure, $80 \%$ have CSA or OSA. ${ }^{14}$ In stable patients with heart failure with both reduced and preserved ejection fraction, the prevalence is about $50 \% .^{2,15}$ As heart failure progress, CSA and complex sleep disorders becomes more prevalent, and likely contribute to the poor diagnosis. ${ }^{2}$

In conclusion, nocturnal sleep disturbances are common in all cardiovascular patients and cause a range of acute and chronic physiological effects that likely contribute to an increased morbidity and mortality. We can expect an increased prevalence of OSA in the future with an ageing and more overweight population. In elderly, the symptoms are often less apparent though OSA is also associated with cognitive impairment. ${ }^{16}$ Despite ample physiological understandings many questions remain, and more evidence particularly on treatment effects is needed before the concept of sleep apnoea as a modifiable risk factor may enter the guidelines. Unfortunately, few such trials are ongoing.

\section{Selected references:}

1. Heinzer R, Vat S, Marques-Vidal P, Marti-Soler $\mathrm{H}$, Andries D, Tobback N, Mooser V, Preisig M, Malhotra A, Waeber G, Vollenweider P, Tafti M and HabaRubio J. Prevalence of sleep-disordered breathing in the general population: the HypnoLaus study. Lancet Respir Med. 2015;3:310-8. DOI: 10.1016/ S2213-2600(15)00043-0

2.Floras JS. Sleep Apnea and Cardiovascular Disease: An Enigmatic Risk Factor. Circ Res. 2018;122:17411764. DOI: 10.1161/CIRCRESAHA.118.310783

3.Dempsey JA, Veasey SC, Morgan BJ and O'Donnell CP. Pathophysiology of sleep apnea. Physiol Rev. 2010;90:47-112. DOI: 10.1152/physrev.00043.2008

4.Spaak J, Egri ZJ, Kubo T, Yu E, Ando S, Kaneko Y, Usui K, Bradley TD and Floras JS. Muscle sympathetic nerve activity during wakefulness in heart failure patients with and without sleep apnea. Hypertension. 2005;46:1327-32. DOI: 10.1161/01.HYP.0000193497.45200.66

6.White LH, Bradley TD and Logan AG. Pathogenesis of obstructive sleep apnoea in hypertensive 
patients: role of fluid retention and nocturnal rostral fluid shift. J Hum Hypertens. 2015;29:34250. DOI: 10.1038/jhh.2014.94

7.Briancon-Marjollet A, Weiszenstein $\mathrm{M}$, Henri M, Thomas A, Godin-Ribuot D and PolakJ. The impact of sleep disorders on glucose metabolism: endocrine and molecular mechanisms. Diabetol Metab Syndr. 2015;7:25. DOI: 10.1186/s13098-015-0018-3

8 .Logan AG, Perlikowski SM, Mente A, Tisler A, Tkacova R, Niroumand M, Leung RS and Bradley TD. High prevalence of unrecognized sleep apnoea in drug-resistant hypertension. J Hypertens. 2001;19:2271-7. DOI: 10.1097/00004872-200112000-00022

9.Bratton DJ, Gaisl T, Wons AM and Kohler M. CPAP vs Mandibular Advancement Devices and Blood
Pressure in Patients With Obstructive Sleep Apnea: A Systematic Review and Meta-analysis. JAMA. 2015;314:2280-93. DOI: 10.1001/jama.2015.16303

10.Becker HF, Jerrentrup A, Ploch T, Grote L, Penzel T, Sullivan CE and Peter JH. Effect of nasal continuous positive airway pressure treatment on blood pressure in patients with obstructive sleep apnea. Circulation. 2003;107:68-73. DOI: 10.1161/01.cir.0000042706.47107.7a

11.Cuspidi C, Tadic M, Sala C, Gherbesi E, Grassi $G$ and Mancia G. Obstructive sleep apnoea syndrome and left ventricular hypertrophy: a meta-analysis of echocardiographic studies. J Hypertens. 2020;38:1640-1649. DOI: 10.1097/ $\mathrm{HJH} .0000000000002435$

FULL REFERENCE LIST

\section{Obstructive Sleep Apnea Management in Patients with Cardiovascular Comorbidities}

\section{DOMINIK LINZ}

Department of Cardiology, Maastricht University Medical Centre and Cardiovascular Research Institute Maastricht,

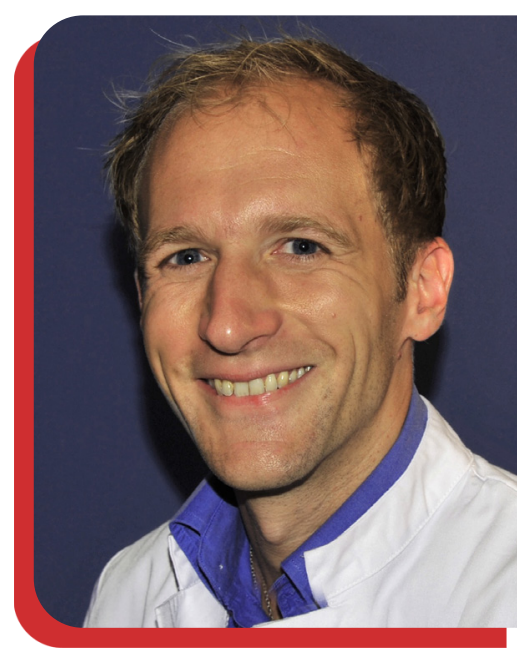

Maastricht, The Netherlands.

DOI:10.30824/2106-15

Obstructive sleep apnea (OSA) is highly prevalent in patients with cardiovascular (CV) comorbidities such as hypertension or atrial fibrillation and is associated with increased risk of mortality, CV events and arrhythmias. ${ }^{1}$ Continuous positive airway pressure (CPAP) is established as first line therapy in OSA patients with moderate or severe OSA due to its impact on both symptoms and quality of life. In clinical care, CPAP is recommended to be combined with lifestyle changes and weight loss.

Up to $50 \%$ of OSA patients may have hypertension, and $30 \%$ of hypertensive patients will likely have OSA. ${ }^{2}$ Patients with untreated OSA followed over 4 years have a 2-3 fold increased risk of developing incident hypertension, independent of the usual risk factors for hypertension. ${ }^{2}$ Metaanalyses of randomized trials of CPAP in patients with hypertension have reported modest blood pressure decreases of between 2 to $3 \mathrm{mmHg} .{ }^{3}$ In patients with atrial fibrillation, the prevalence of OSA in patients with AF is higher (21-74\%) than in controls without AF (3-49\%). ${ }^{4}$ In a meta-analyses of several non-randomized studies, the use of CPAP was associated with a $42 \%$ decreased risk of AF-recurrence. ${ }^{5}$ Randomized studies in the field of atrial fibrillation management are currently ongoing.

Three randomized controlled trials assessed the effects of CPAP on secondary prevention of CV events (Table). The Sleep Apnea Cardiovascular Endpoints (SAVE) trial randomised over 2000 